\title{
An atypical presentation of necrotising fasciitis
}

\author{
K Kunz ${ }^{1}$, F Seidel ${ }^{1}$, L Weidhase ${ }^{1}$, P Hepp ${ }^{2}$, S Strocka ${ }^{3}$, T Gradistanac ${ }^{4}$, S Petros ${ }^{1}$ \\ ${ }^{1}$ Medical Intensive Care Unit, University Hospital Leipzig, Germany \\ ${ }^{2}$ Department of Orthopaedics, Trauma and Plastic Surgery, University Hospital Leipzig, Germany \\ ${ }^{3}$ Department of Radiology, University Hospital Leipzig, Germany \\ ${ }^{4}$ Institute of Pathology, University Hospital Leipzig, Germany
}

\section{ABSTRACT}

Necrotising fasciitis is a rare infection with a high mortality rate. Clinical manifestation may be influenced by an underlying disease state. We report a case of a 70-year-old man with an anaplastic large-cell lymphoma who presented with inconclusive signs of necrotising fasciitis following a neutropenic phase after chemotherapy. Surgical exploration did not reveal the typical macroscopic features of necrotising fasciitis. Microbiological investigations revealed Escherichia coli. The lack of massive tissue inflammation, probably due to the neutropenia in our patient, has not been described by other authors. Diagnostic uncertainties owing to masked or uncharacteristic signs in immune compromised patients may lead to a delayed surgical debridement, of which clinicians should be aware.

\section{KEYWORDS}

Necrotising Fasciitis - Lymphoma - E.Coli - MTX - Neutropenia

Accepted 24 October 2016

CORRESPONDENCE TO

Sirak Petros, E: sirak.Petros@medizin.uni-leipzig.de

\section{Case report}

A 70-year-old man was admitted to our hospital with impaired vision and a downbeat nystagmus. He had a history of anaplastic large-cell lymphoma that was in remission following chemotherapy. However, magnetic resonance imaging (MRI) and a spinal tap on admission confirmed the clinical suspicion of a lymphomatous meningitis. Intrathecal followed by systemic high-dose methotrexate $(6640 \mathrm{mg}$ ) was administered on day 16 after admission. The patient developed neutropenia on day 20. A mild swelling of the left thigh, with marked pain, fever, tachycardia and tachypnoea were observed on day 23 , and the patient was transferred to the intensive care unit (ICU). Laboratory workup revealed a serum C-reactive protein of $438 \mathrm{mg} / \mathrm{l}$ and procalcitonin of $33.7 \mathrm{ng} / \mathrm{ml}$. Maximum blood lactate in the first 24 hours after ICU admission was $2.9 \mathrm{mmol} / \mathrm{l}$.

An MRI of the left leg showed a slight oedema of the gastrocnemius muscle with no pathological enhancement of the fascia (Fig 1). Antibiotic therapy with imipenem/cilastatine was started and a senior surgeon was immediately consulted, because of the high suspicion of necrotising fasciitis. Surgical exploration of the left leg revealed no typical macroscopic signs of necrotising fasciitis. Only slight bleeding in the soleus and flexor hallucis longus muscles was observed. Tissue biopsy and smears were sent for diagnostic workup and a primary wound closure was performed as the macroscopic features made necrotising fasciitis seem unlikely. Histology showed inflammation and necrosis of the superficial fascia with polymorphonuclear cell infiltration highly suspicious of necrotising fasciitis (Fig 2). Fibrinoid thrombi and necrosis of vessel walls were not observed.

The microbiological workup revealed growth of Escherichia coli in the biopsy specimens and the blood cultures. These findings, together with the continuing systemic inflammation, warranted a surgical second look, which was carried out on day 25. Once again, no suspicious signs of necrotising fasciitis were found. Nevertheless, the medial parts of the fascia were excised and the surgical wound was closed. A radical removal of the infected tissue was not certain because of the missing tissue boundaries between the healthy and infected parts. A radical debridement of the lateral compartment was not done, owing to the lack of evidence of infection in the initial smear, to avoid a possibly unnecessary surgical trauma in a neutropenic patient.

The condition of the patient further deteriorated, with an extension of the swelling to the left hip. He became confused and restless with involuntary movements. A repeat spinal tap revealed lymphoma cells. Owing to the intractable lymphomatous meningitis and the multiple organ dysfunction syndrome, the management goal was changed in accordance with the patient's wishes to palliative care and the patient died on day 33. The family did not consent to an autopsy, which would have been helpful to identify the extent of the disease.

Necrotising fasciitis is a rare but life-threatening infection with a mortality rate of $50-75 \% .^{1,2}$ It is characterised by 


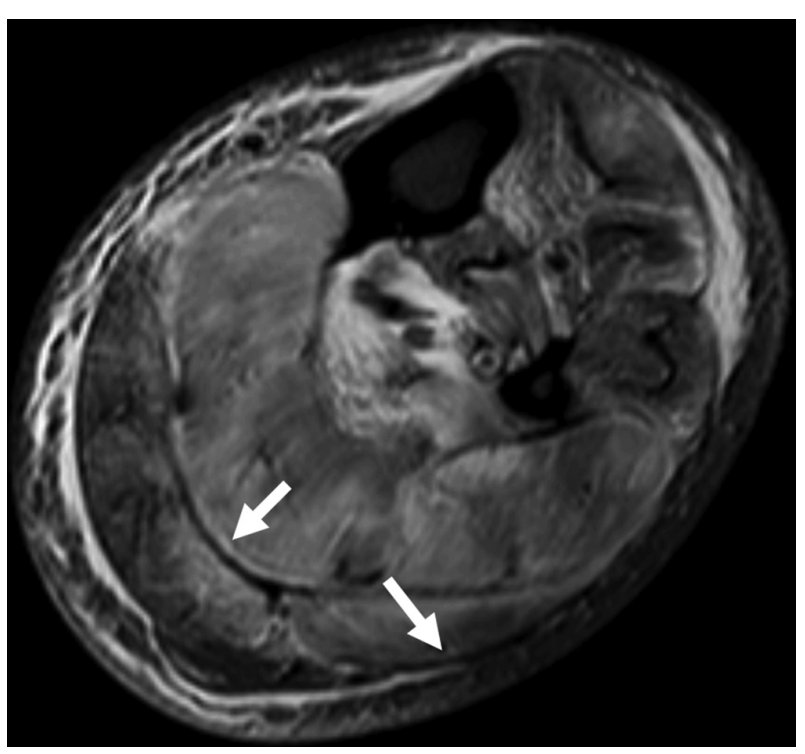

Figure 1 Magnetic resonance image (T2) of the left thigh, showing marked oedema of the gastrocnemius muscle and subcutaneous tissue but normal signals of the fascia (arrows)

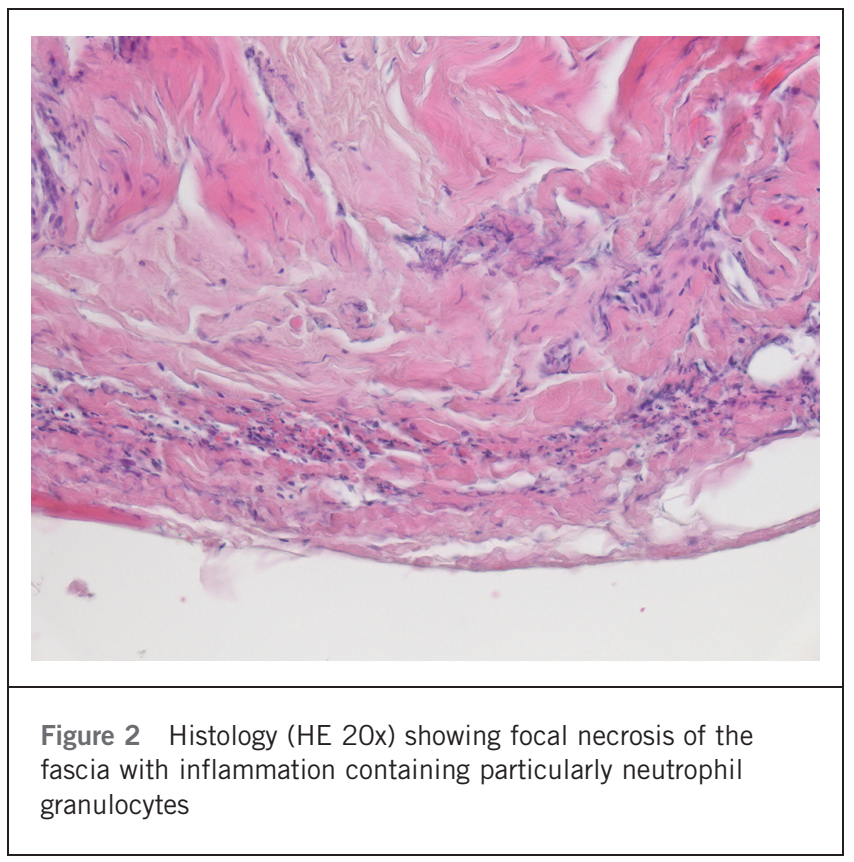

rapid tissue destruction, with the development of a systemic inflammation and multiple organ dysfunction syndrome. Smoking, obesity, diabetes mellitus and immunocompromise are prevalent among these patients. ${ }^{3}$ The typical clinical sign of necrotising fasciitis is the extreme and disproportional pain contrasting with slight dermal signs. This can lead to misdiagnosis in the early stages of the disease and may cause a delay in life-saving surgical debridement and antibiotic therapy. ${ }^{1}$ The distinction of necrotising fasciitis from other soft-tissue infections is still a diagnostic challenge. The Laboratory Risk Indicator for Necrotizing Fasciitis (LRINEC) score showed a positive predictive value of $92 \%$ (95\% confidence interval $84.2-96.0$ ) for identifying necrotising fasciitis in one cohort. ${ }^{4}$ One case report in a patient with leukaemia suggested that the LRINEC score may be helpful in an equivocal case of necrotising fasciitis. ${ }^{5}$ However, there have been no prospective trials validating this score. In our patient, although this score was 12 , which is high, it may be biased because of pathological findings related to the underlying malignant disease. Marked elevation of inflammation markers and blood lactate, as has been observed in our patient, can be signs of a deteriorating course, but they are not specific to necrotising fasciitis. Other authors have proposed an improvement of the score with more emphasis on the 'pain out of proportion'. ${ }^{6}$ The reported high sensitivity of this score has also been questioned by others, ${ }^{3,7}$ giving the macroscopic and histological picture more relevance. ${ }^{7}$ In our patient, the massive necrosis typical of necrotising fasciitis was not evident. However, the histological findings may have been influenced by the underlying disease and the chemotherapy.

In conclusion, necrotising fasciitis is a rare disease with a high mortality rate, particularly in immune compromised patients. The surgical treatment of a clinically highly probable necrotising fasciitis but intraoperative unsuspicious findings in patients with an underlying hematologic malignancy remains uncertain.

\section{References}

1. Morgan MS. Diagnosis and management of necrotising fasciitis: a multiparametric approach. J Hosp Infect 2010; 74(4): 249-257.

2. Misiakos EP, Bagias G, Patapis $P$ et al. Current concepts in the management of necrotizing fasciitis. Front Surg 2014; 1: 36.

3. Hodgins N, Damkat-Thomas L, Shamsian N et al. Analysis of the increasing prevalence of necrotising fasciitis referrals to a regional plastic surgery unit: a retrospective case series. J Plast Reconstr Aesthet Surg 2015; 68(3): 304-311.

4. Wong C-H, Khin L-W, Heng K-S et al. The LRINEC (Laboratory Risk Indicator for Necrotizing Fasciitis) score: a tool for distinguishing necrotizing fasciitis from other soft tissue infections. Crit Care Med 2004; 32(7): 1,535-1,541.

5. Song CT, Hamilton R, Song $C$ et al. Enabling the diagnosis of necrotising fasciitis without associated skin changes in a paediatric patient with acute Iymphoblastic leukaemia: the LRINEC score. J Plast Reconstr Aesthet Surg 2015; 68(1): e23-e24

6. Borschitz T, Schlicht S, Siegel E et al. Improvement of a clinical score for necrotizing fasciitis: 'pain out of proportion' and high CRP levels aid the diagnosis. PloS One 2015; 10(7): e0132775.

7. Breakey W, Morrison R, Rahman K Re: Enabling the diagnosis of necrotising fasciitis without associated skin changes in a paediatric patient with acute lymphoblastic leukaemia: The LRINEC score. J Plast Reconstr Aesthet Surg 2015; 68(7): 1,016-1,017 